\title{
Análisis de los sitios web del movimiento por los derechos sexuales y reproductivos en México
}

\author{
Yin-Zu Chen \\ Doctora en Sociología (Ruhr-Universität Bochum) \\ Profesora en la Universidad Nacional de Taipei, Taiwan \\ chenyinzu@gmail.com
}

\begin{abstract}
Resumen El artículo analiza los sitios web del movimiento por los derechos sexuales y reproductivos en la Ciudad de México con el objetivo de explicar la discrepancia entre la potencialidad del internet y su uso real; y analizar cómo el contexto sociopolítico influye sobre las estrategias de las organizaciones de los movimientos sociales basadas en los medios digitales. El diseño de investigación consta de dos procedimientos: el análisis de los 20 sitios web de las organizaciones y entrevistas semi-estructuradas con 19 activistas. El resultado muestra que aparte de las características organizativas, el conocimiento sobre la tecnología informática en general, la burocracia en el proceso de financiamiento y la relación con los medios tradicionales de comunicación, son factores que intervienen en el uso de Internet por parte de las organizaciones de derechos sexuales y reproductivos en México.
\end{abstract}

Palabras-clave: internet, movimiento social, derechos sexual y reproductivos, México.

\section{Introducción}

L A APROBACIÓN DE LA DESCRIMINALIZACIÓN DEL ABORTO en el Distrito Federal, México, en el 2007 provocó una intensa movilización de las organizaciones civiles en contra del aborto. Éstas exigieron al gobierno otorgar al feto el estatus legal de persona mediante una reforma constitucional y con ello evitar la posible legalización sucesiva del aborto en otros Estados. Mientras tanto, las organizaciones y grupos a favor de los derechos sexuales y reproductivos se movilizaron para la provisión de servicios abortivos en el D.F.. Varias organizaciones y grupos en la Ciudad de México han participado en esta guerra del aborto; también en esta ocasión, académicos, políticos, profesionistas y feministas luchan por la defensa de los derechos sexuales y la libertad reproductiva (Kulczycki, 2007).

Un fenómeno notable en esta lucha es la extensión de las acciónes colectivas hacia el espacio virtual: el incremento de la presencia de los videos en línea, sitios web y listas de correo electrónico donde se discuten sobre el aborto y las acciones que llevan a cabo. Internet, como una herramienta de comunicación, facilita la planeación de acciones colectivas, ayuda a los ciudadanos que apoyan al movimiento a construir una comunidad. A largo plazo Internet apoya a las protestas y movilizaciones en el mundo real y el mundo virtual (Graber et al., 2004). 
Este estudio analiza los sitios web de los grupos y organizaciones del movimiento por los derechos sexuales y reproductivos como parte de una estrategia de movilización por Internet. El objetivo es explicar la discrepancia entre la potencialidad del Internet y su uso real por las organizaciones de movimientos sociales, así como identificar los factores contextuales que intervienen a las acciones virtuales.

El argumento principal de este artículo es que, además de las características organizacionales, los factores contextuales como 1) la familiaridad con las técnicas para diseñar sitios web, 2) la complejidad del procedimiento para recaudar fondos externos y 3) la relación entre las organizaciones y los medios tradicionales de comunicación son relevantes para entender el uso del Internet por el movimiento por los derechos sexuales y reproductivos en la ciudad de México.

\section{El uso de Internet por los movimientos sociales}

Con el incremento del índice de penetración de Internet, la utilización de las nuevas tecnologías de información se ha vuelto una práctica cotidiana para los activistas de los movimientos sociales, y al mismo tiempo Internet se ha transformado en una herramienta estratégica de movilización. Un caso conocido y ampliamente estudiado es la lucha del Ejército Zapatista de Liberación Nacional en Chiapas. La resistencia indígena contra la represión del gobierno en el sudeste del país alcanzó la audiencia internacional cuando utilizó Internet como instrumento de interconexión y protesta (Froehling, 1997; Schulz, 2007). Las posibilidades que ofrecía la nueva tecnología a las protestas locales y grupos margiales captaron la atención de los investigadores.

Al comienzo del estudio sobre las estrategias de Internet de los movimientos sociales en 1990, los investigadores consideraron a Internet como un instrumento que empodera a los grupos marginales y movimientos sociales. La transmisión de información con alta velocidad y su bajo costo posibilitan la expresión libre de opiñones y organizaciónes de acciones colectivas, de esta manera facilitan la distribución del poder en la esfera pública y otros dominios (Bennett, 2003).

Sin embargo esta afirmación contradice los resultados de los estudios sobre la "brecha digital" que investiga el acceso diferencial a la tecnología digital entre los grupos de distintas posiciones sociales. El punto central de estos estudios considera que la falta de infraestructura y el nivel educativo insuficiente dan origen a la desigualdad en el uso de la tecnología. Desde este punto de vista, los grupos marginales tienen menos posibilidad de aprovechar las ventajas que ofre Internet. El análisis de Beckles (2001) muestra una reconcentración del poder en el ciberespacio en cuantoa a la clase social y la etnicidad. Este resultado pone en evidencia que el uso diferencial de la tecnología digital refleja las relaciones de poderes en el mundo real.

Tanto el aprecio entusiasta sobre la utilidad que provee Internet en la lucha de los grupos sociales marginales y el punto de vista pesimista de los estudios sobre la brecha digital, los estudios empíricos desde ambas perspectivas revelaron que la diferencia del uso de la tecnología digital no puede atribuirse a una sola explicación, tal como la inexistencia de la infraestructura apropiada o el poco accesibilidad de Internet. Por ejemplo, Stein et al. (2009) examinaron la construcción de los sitios web de las organizaciones sociales en los Estados Unidos y mostraron que la mayoría de estas organizaciones no utilizaba la web a su máximo potencial. Esta evidencia mostró que no todas las organizaciones conocían los posibles usos que Internet ofrece, y tampoco los ponían en práctica incluso si los conocían. Por qué esta discrepacia entre la potencialidad de Internet y su uso real y cómo se explica los usos diferentes por las organizaciones de movimientos sociales son preguntas centrales de aborda el presente trabajo.

Además de la infraestructura de la tecnología y el acceso a Internet se debe considerar otros factores para analizar cómo se utiliza la nueva tecnología de comunicación. Como señalan Ganesh y Barber (2009), el análisis del uso que se le da a Internet necesitaba reconocer "otras influencias sociopolíticas, espacios, interacciones y eventos; otras fuentes de pericia, conocimiento y recursos" (Ganesh y Barber, 2009, p. 260).

Dos enfoques existen en los estudios actuales sobre el uso que los movimientos sociales de Internet: uno explora esta cuestión desde una perspectiva racional-instrumentalista y toma a las características de la organización como causas principales; el otro considera que el uso de la tecnología está a función de los factores externos basados en el contexto donde el movimiento social está activo.

Dentro de la perpectiva racional-instrumentalista se encuentra el estudio de Mario Diani (2001), cual demostró que el uso de Internet depende de las metas de las organizaciones sociales. Las que pretendían articular los recursos profesionales podrían estar motivadas a utilizar Internet para difundir la información en línea, pero rara vez utilizan la red como una herramienta de comunicación para incrementar la interacción entre los líderes y los miembros, pues la creación de lazos con los grupos de base no es su 
prioridad. Este resultado sustenta a la perspectiva del comportamiento racional de la organización social como un usuario activo que aplica la nueva tecología según sus propósitos.

Un estudio más detallado en esta perspectiva sobre el uso racional de Internet es el análisis de las organizaciones de mujeres holandesas hecho por Arthur Edwards (2004). En este estudio, doce organizaciones fueron seleccionadas y clasificadas según su función principal dentro de del movimiento en cuatro tipos: las organizaciones de los movimientos sociales, las coaliciones, las organizaciones de apoyo y las organizaciones representativas. Edwards argumentó que las diferentes metas y actividades de las organizaciones "reales" determinaban el diseño de su sitio web. Las organizaciones de movimientos - los organizadores principales de las protestas - incluyeron un foro de movilización y comunicación en sus sitios web, mientras que los sitios de las coaliciones se focalizaron en la formación de redes y en la construcción de la identidad colectiva de los movimientos sociales. Comparado con las organizaciones de apoyo, que usan sus sitios web como medios de autorepresentación y de difusión de la información sobre los temas principales, los sitios web de las organizaciones representativas buscaban el apoyo y la comunicación interna del movimiento.

Estos estudios van más allá del simple determinismo tecnológico y colocan la racionalidad de los actores, en este caso las organizaciones, en el centro del análisis. Al explicar el uso de Internet en relación con las características organizacionales, se asumieron que: 1) la posibilidad de accesar a Internet es la misma para todos los movimientos sociales y sus integrantes, 2) la organización de los movimientos sociales siempre se comporta según sus cálculos racionales. Este enfoque se centra en los determinantes internos de una organización, pero ignora el razonamiento del actor se encuentra influenciado por su contexto sociopolítico, lo cual difiere entre las localidades geográficas y entre grupos sociales.

Respecto a la pregunta sobre qué tanto influyen el contexto y el ambiente del movimiento social en el diseño y uso del sitio web, Pudrovska y Ferree (2004) compararon los sitios web de organizaciones sociales trasnacionales en Europa y en los Estados Unidos. Ellas llegaron a la conclusión de que el discurso del sitio web de European Women's Lobby reflejaba la estructura de oportunidad política de la Union Europea. Mientras Pudrovska y Ferree (2004) investigaron el uso de Internet en función del contexto político, Della Porta y Mosca (2009) propusieron otras dos condiciones contextuales que influenciaban el uso de Internet: la posibilidad de acceso a este medio y la constelación del movimiento (si es polarizado o integrado). Según su estudio, estas dos características contextuales tienen una infuencia significativa en las cualidades democráticas de los sitios web, tales como la provisión de información y la transparencia en cuanto a la cuestión financiera.

Este enfoque contextual amplía el análisis del uso de Internet desde la perspectiva racional-instrumentalista de la organización hacia el medio ambiente donde se localiza la organización. Hay varios factores del contexto que influyen el comportamiento y decisión de una organización social sobre su uso de la tecnología, puede referirse a la estructura política, la infraestructura de Internet, la constelación del movimiento social etc. Cada movimiento social tiene su propio contexto y, además, cada sociedad o localidad ofrece diferentes factores contextuales a los movimeintos sociales.

Este artículo busca especificar los factores contextuales que influyen sobre los sitios web del movimiento de derechos sexuales y reproductivos en la Ciudad de Mexico mediante el uso de ambos enfoques presentados anteriormente. Se entienden que los factores contextuales influenciyen las características y conductas organizacionales, que a su vez afectan la construcción de sus representaciones virtuales en Internet. Al considerar que los sitios web de las organizaciones son resultados del contexto y las características de la organización, este análisis muestra que más allá de las influencias de las características organizacionales, los sitios web de este movimiento difieren de los sitios de las organizaciones trasnacionales estadounidenses o europeas debido a tres factores de contexto específicos: el conocimiento que se tiene sobre Internet, el proceso para la obtención de apoyo financiero internacional y la estructura de los medios tradicionales. A continuación se presenta brevemente el movimiento estudiado.

\section{El movimiento por los derechos sexuales y reproductivos en México: una historia breve}

La autonomía reproductiva y sexual fue y sigue siendo un tema crucial del movimiento feminista mexicano. La primera publicación feminista en México en 1979 se tituló "Maternidad voluntaria: una guía de métodos anticonceptivos", y cuestionaba la prohibición de los anticonceptivos y el control de la sexualidad femenina (Ortiz-Ortega, Barquet, 2010).

El tema de la autodeterminación sexual y reproductiva se desarrolló de forma paralela en México con los movimientos feministas en los Estados Unidos y Europa. La fundación de la organización feminista pionera dedicada a la salud reproductiva de las muje- 
res, Comunicación, Intercambio y Desarrollo Humanos en América Latina (CIDHAL), en Morelos en 1969, fue con el apoyo de The Boston Women's Health Collective y la Federation of Feminist Women's Health Centers en los Estados Unidos (Cardaci, 2004). Esta fue la primera organización mexicana que promovió la salud y los derechos humanos entre las mujeres de bajos ingresos para lograr su empoderamiento.

La lucha del movimiento feminista a favor de la decisión de la mujer sobre su cuerpo y reproducción se tradujo en el interés del gobierno en reducir el crecimiento de la población al hacer que los anticonceptivos estuvieran disponibles en México a través de la introducción de las políticas de población y el programa de planificación familiar en 1974. Hubo dos críticas principales a esta política que originaron la movilización feminista: este programa político relacionado con el cuerpo de la mujer estaba delineado por hombres y entre sus medidas no consideraba la legalización del aborto.

Junto a la demanda sobre la legalización del aborto durante los años 1970s, los grupos y organizaciones feministas exigieron la separación de la sexualidad y la reproducción así como la libertad de decidir el número de niños a concebir. Ellas denunciaron la aplicaciónn de métodos anticonceptivos inseguros sin el consentimiento de las mujeres y apoyaron a las víctimas femeninas de la violencia.

Estos temas sobre los derechos sexuales y reproductivos tuvieron contiuidad mediante las actividades feministas en los 1980s. La institucionalización de los grupos feministas en ONGs floreció con el incremento de financiamiento internacional de desarrollo para México. La maternidad libre y voluntaria, la libertad sexual y la lucha para acabar la violencia contra las mujeres se volvieron los temas centrales de los programas de las ONGs feministas.

Una serie de conferencias internacionales organizadas por las Naciones Unidas en los años 1990s - especialmente la Conferencia Internacional de Desarrollo y Población en El Cairo en 1994 y la Conferencia Mundial de la Mujer en Beijin en 1995 - construyeron un contexto favorable para el establecimiento de la red feminista mexicana. Las conferencias preparatorias nacionales o regionales juntaron a los grupos locales y ONGs feministas para articular sus exigencies y formular un programa estratégico. Este período se caracterizó por la expansión nacional e internacional de las redes feministas y por el intercambio intenso entre las luchas feministas.

La fundación de la Red por la Salud de la Mujer en el Distrito Federal en 1993 - de un año antes de la conferencia de El Cairo - fue un paso hacia la coor- dinación de las actividades y la acción colectiva del movimiento. Una de las actividades de la red era proporcionar información sobre los derechos sexuales y reproductivos a las mujeres embarazadas y al público en general. La creación sucesiva de las coaliciones y redes ${ }^{1}$ que cubría este tema formó una articulación de acción intensa a nivel nacional (Cardaci, 2004: 86).

Después de las conferencias mundiales, el monitoreo de la implementación nacional y local de los programas políticos internacionales acordados - el Consenso de El Cairo y la Plataforma para la Acción de Beijin - llevó a una mayor participación feminista en el dominio institucional y en la esfera pública. Los derechos sexuales y reproductivos se volvieron presentes en la agenda política al mismo tiempo, las organizaciones feministas expandieron sus actividades de promoción de los derechos reproductivos y sexuales hacia otros grupos sociales: hombres y adolescentes.

Una nueva movilización por la legalización del aborto comenzó en 1999, cuando la víctima de violación de 13 años, Paulina, fue obligada a dar a luz en Baja California. En el 2000, el gobierno del D.F. extendió los motivos para el aborto legal: cuando la salud de la mujer estaba en peligro, cuando el feto sufría de malformaciones y cuando la inseminación artificial no consensual ocurría. Desde 2007, con la aprobación de la descriminalización del aborto, se introdujeron legalmente en el D.F. los servicios que ofrecen esta práctica a cualquier mujer que lo solicite, dentro de las primeras doce semanas de embarazo. Al mismo tiempo, las fuerzas conservadoras y religiosas iniciaron un contra-movimiento para impedir la legalización del aborto en otros Estados de la República y pidieron que se incluyera en la constitución la definición del feto como una persona con derecho a la protección legal.

\section{Datos y procedimientos}

El diseño de la investigación consiste en dos procedimientos: el análisis de los sitios web y entrevistas con los activistas. Hay dos razones para utilizar a los sitios web como datos de la investigación para el estudio del uso de Internet por los movimientos sociales: primero, el sitio web es la forma principal de autorepresentación en el mundo virtual, una organización puede transmitir su identidad, sus metas y presentar sus relaciones en el mundo virtual. Segundo, el sitio web puede diseñarse para apoyar a las actividades del movimiento social y de la organización, por ejemplo, puede servir como un foro de la comunicación inter- 
na, como medio de llamado a acciones, difundir noticias o como instrumento de recaudación de fondos. El análisis de los sitios web nos permite captar el razonamiento y el uso de Internet por cada organización.

La selección de la muestra se inicia con la lista de los integrantes de la Red Democracia y Sexulidad (DEMYSEX), una red nacional de organizaciones a favor de los derechos sexuales y la salud reproductiva. Luego de buscar las organizaciones por Internet resulta que dentro de 243 organizaciones y grupos en todo el país, 29 de ellos se encuentran en la Ciudad de México, de las cuales, 16 poseen sitios web propios. A través de los links de los sitios web de estas organizaciones se encontraron otros grupos y organizaciones del movimiento que no están registradas en la lista de DEMYSEX. Incluyendo a éstas, se consideraron para el presente análisis veinte sitios web de grupos y organizaciones en la Ciudad de México que participan de alguna forma en el movimiento de los derechos sexuales y reproductivos. Los sitios web fueron descargados con el software Teleport, fueron clasificados y codificados de acuerdo a las categorías requeridas para la investigación.

Para entender el razonamiento de los activistas en la construcción de su sitio web, diescinueve miembros de ocho organizaciones y grupos en la Ciudad de México fueron entrevistados de manera individual en febrero y agosto de 2009. El objetivo era entender los factores contextuales que afectaron la desición de la organización cuando crearon y diseñaron sus sitios web.

\section{Mapeo de los sitios web del movimiento por los derechos sexuales y reproductivos}

Para analizar las influencias de las características organizacionales en el diseño de los sitios web, se clasificaron los grupos y organizaciones estudiados de acuerdo a dos criterios: el papel que desempeña dentro del movimiento y el nivel organiativo (incluyendo la estabilidad de los fondos económicos y la estructura interna). Estos dos criterios están relacionadas entre sí: el primero describe la relación entre los grupos y organizaciones dentro del movimiento, el segundo se refiere a las diferencias de los recursos y la estructura interna entre las organizaciones.

De esta clasificación resulta cuatro tipos de organizaciones: (a) los grupos de protesta: grupos pequeños y de creaciónes recientes que participan activamente en las acciones de protesta, (b) las organizaciones de los movimientos sociales: organizaciones establecidas con una estructura formal, principalmente represen- tan al movimiento en las negociaciones con el gobierno, (c) coaliciones: redes y enlaces de grupos y organizaciones para determinada tema, (d) organizaciones de apoyo: organizaciones con estructura más rígida y con mayores recursos que brindan servicios a la comunidad del movimiento social o al público en general. Deacuerdo a esta tipología hay cinco coaliciones, cuatro grupos de protesta, cinco organizaciones de movimientos sociales y seis organizaciones de apoyo entre las veinte organizaciones y grupos seleccionados para este estudio (ver Cuadro 1).

Se codifica a las herramientas y tareas presentadas en los sitios web para analizar el diseño de cada sitio web y para explicar las diferencias en su uso. Según estudios existentes, Internet presentan las siguientes funciones para los movimientos sociales: difundir la información, solicitar opiniones, discusión, reclutamiento, creación de una red y facilitamiento del contacto interno, movilización y servicio (Edwards, 2004). Internet es también vista como una alternativa para difundir noticias alternativas, crear esferas públicas virtuales y organizar la acción política colectiva (Rheingold 2003). Della Porta y Mosca (2009) resumieron cinco dimensiones del sitio web: información a la opinion pública, organización en línea, protesta, construcción de la identidad y transparencia.

Se observó que en los sitios de las organizaciones mexicanas la función de la protesta virtual sólo estaba presente en el sitio de una organización - la portal de una coalición: Tú puedes salvar la Vida. La creación de una esfera pública virtual sólo se encontraba en el grupo de protesta, Decidir. Este hecho demuestra que el carácter interactivo de la comunicación digital está subdesarrollado en los casos mexicanos. Como concluyó Ligia Tavera Fenollosa (2001), las ONGs mexicanas usan Internet como un medio de comunicación (como el correo electrónico) alternativo al teléfono o el fax, y la función de Internet mmas bien para recibir información y no para generar foros electrónicos. Aunque el uso de Internet podría ayudar a las ONGs a llevar a cabo su tarea de una forma más eficiente y económica, las organizaciones mexicanas no exploran la posibilidad de crear un nuevo espacio en el mundo vistual para discutir o deliberar.

Despúes de considerar las funciones existentes de los sitios web de las organizaciones de movimientos sociales mexicanos, los siguientes funciones de sitios web se consideraron para este estudio: (a) comunicación: si los sitios sirven para la comunicación entre los miembros (interno) o no, y si tienen espacio para que los visitantes dejen mensajes y un foro para facilirar la comunicación (externo) o no; (b) creación de redes de contacto: si los sitios proveen ligas a otros sitios o no; (c) llamado a la acción: si los sitios web brindan información sobre las acciones o actividades de su propia organización (sus actividades) o activi- 
dades de otras organizaciones; (d) noticias: si los sitios web presentan noticias actuales relacionadas con los temas de sexualidad y reproducción; (e) información: si los sitios ofrecen información relacionada con los derechos sexuales y reproductivos o no; (f) recaudación de fondos en línea: si las organizaciones utilizan Internet como instrumento para recolectar recursos e integrar una liga para las donaciones en línea; (g) contabilidad: si los sitios son considerados como un instrumento de transparencia al presentar un reporte financiero en las páginas virtuales de la organización (ver cuadro 1).

Las tres funciones más frecuentes en los sitios web de las organizaciones estudidas son: creaciones de redes de contactos (enlaces), movilizar a las personas para que participen en actividades (llamado a la acción) y proveer información sobre los derechos sexuales y reproductivos, incluyendo servicios de salud, métodos anticonceptivos o la práctica del aborto (información). Todas estas funciones se presentan en catorce de los veinte casos.

Las funciones menos presentes son: diseñar el sitio web para movilizar los recursos financieros (recaudación de fondos en línea) y reportar la situación financiera de la organización (contabilidad). La primera sólo está disponible en el sitio de dos organizaciones y la última en el de tres.

En resúmen, Internet no funciona como un instrumento de comunicación interactiva y tampoco como una esfera pública alternativa para el movimiento de derechos sexuales y reproductivos en la Ciudad de Mexico. La utilización de la nueva tecnología en este caso va dirigida principalmente a informar al público en general, o a participantes poten- ciales sobre las actividades de protesta y sobre otras organizaciones en la comunidad del movimiento.

\section{¿lmportan las características organizaciona- les?}

¿Hasta qué grado las características organizacionales influyen sobre el diseño del sitio web, tal como lo supone la perspectiva racional-instrumentalista? El presente análisis muestra que la mayoría de las coaliciones (4/5), grupos de protesta (3/4) y organizaciones de movimientos sociales $(5 / 5)$ hacen llamados a la acción en sus sitios web. Comparado con ellos, tres de seis organizaciones de apoyo no anuncian protestas en Internet porque participación en acciones colectivas no forma parte de sus actividades principales. Por otra parte, las funciones para recaudar los recursos financieros y materiales $(2 / 6)$ y el promover la transparencia entre la sociedad civil (3/6) sólo están disponibles en los sitios de las organizaciones de apoyo.

Estos resultados confirman que el rol que desempeña la organización dentro del movimiento explica el diseño de los sitos. Cuando los grupos u organizaciones ven la movilización colectiva como una de sus actividades principales, Internet se utiliza como un instrumento para difundir el llamado a las actividades colectivas. La misión de las organizaciones de apoyo - que tienen más recursos financieros y estructuras formales - reside en proveer recursos para apoyar a otras organizaciones o para brindar servicios de salud en las comunidades, consecuentemente, utilizan sus sitios web para la recaudación de fondos. Por ejemplo, Semillas - Sociedad Mexicana Pro Derechos de la Mujer, A. C. - es una organización no lucrativa que financía

Cuadro 1: Tipos de organizaciones y las funciones de sus sitios web

\begin{tabular}{|l|c|c|c|c|c|}
\hline & $\begin{array}{c}\text { Coaliciones } \\
(5)\end{array}$ & $\begin{array}{c}\text { Grupos de } \\
\text { protesta }(4)\end{array}$ & $\begin{array}{c}\text { Organizaciones } \\
\text { de movimientos } \\
\text { Sociales } \\
(5)\end{array}$ & $\begin{array}{c}\text { Organizaciones } \\
\text { de apoyo ( 6) }\end{array}$ & Total \\
\hline Comunicación (interna) & $(2 / 5)$ & $(3 / 4)$ & $(0 / 5)$ & $(1 / 6)$ & $(6 / 20)$ \\
\hline Comunicación (externa) & $(2 / 5)$ & $(4 / 4)$ & $(1 / 6)$ & $(9 / 20)$ \\
\hline Enlaces & $(3 / 5)$ & $(4 / 4)$ & $(5 / 5)$ & $(2 / 6)$ & $(14 / 20)$ \\
\hline $\begin{array}{l}\text { Llamado a acción } \\
\text { (actividades propias) }\end{array}$ & $(4 / 5)$ & $(3 / 4)$ & $(5 / 5)$ & $(1 / 6)$ & $(12 / 20)$ \\
\hline $\begin{array}{l}\text { Llamado a acción } \\
\text { (actividades organizadas por otros) }\end{array}$ & $(2 / 5)$ & $(4 / 4)$ & $(5 / 5)$ & $(2 / 6)$ & $(12 / 20)$ \\
\hline Noticias & $(3 / 5)$ & $(2 / 4)$ & $(4 / 5)$ & $(5 / 6)$ & $(14 / 20)$ \\
\hline Informaciones & $(0 / 5)$ & $(0 / 5)$ & $(0 / 5)$ & $(2 / 6)$ & $(2 / 20)$ \\
\hline Recaudación de fondo & $(0 / 5)$ & $(0 / 5)$ & $(0 / 5)$ & $(3 / 6)$ & $(3 / 20)$ \\
\hline Contabilidad & & $(2 / 4)$ & $(5)$ & $(20)$ \\
\hline
\end{tabular}


a otros grupos y organizaciones para la promoción y ejercicio de los derechos de la mujer. La Fundación Mexicana para la Planeación Familiar A.C. (MEXFAM) se dedica al servicio comunitario a brindar apoyo médico para la salud reproductiva. Para ambas organizaciones, los sitios web son espacios públicos donde pueden presentar sus reportes financieros y así mantener su contabilidad y legitimidad.

Sin embargo, algunos resultados del Cuadro 1 contradicen la afirmación de que los sitios web están diseñados a función de las características organizacionales: primero, la construcción de una comunidad del movimiento y la construcción de la identidad colectiva entre sus miembros es crucial para una organización y movimiento social, pero la mayoría de los sitios no tienen las herramientas para la comunicación entre sus miembros (interna) o entre la organización y el público (externa). Sólo dos de cinco coaliciones tienen la herramienta interactiva de comunicación y esto contradice la conclusión de Edwards (2004) que dice que una coalición usaría Internet para la creación de redes de contacto, el intercambio y la construcción de una comunidad. Al mismo tiempo, todos los sitios de los grupos de protesta ofrecen un espacio para la interacción.

Segundo, doce de las veinte organizaciones estudiadas en la Ciudad de México publicaron noticias relacionadas al tema de derechos sexuales y reproductivos en sus sitios que ya se habían presentado en los medios masivos de comunicación tradicionales (periódico, radio o televisión), tales como declaraciones de políticos sobre la legalización del aborto, el nuevo programa de salud reproductiva en la Ciudad de México etc. Publicar noticias en los sitios es un uso común para las organizaciones mexicanas, pero es menos usual en los sitios de las organizaciones de los países industrializados.

Las dos observaciones descritas anteriormente requieren explicaciones más allá del determinismo de las características y racionalidad de las organizaciones sobre el uso de la internet. Los datos cualitativos recolectados mediante entrevistas con los miembros de estas organizaciones revelan que la motivación, las decisiones sobre la creación de sitios y su diseño son influenciados por tres factores contextuales: el relativamente alto costo del conocimiento de la tecnología de la comunicación digital, el apoyo financiero externo y el apoyo de los medios tradicionales de comunicación al movimiento.

\section{El conocimiento sobre la tecnología digital de comunicación}

Casi la mitad de los usuarios de Internet (46\%) en México se concentran el la Ciudad de México. Sólo el 37\% de los usuarios de Internet en el país tiene acceso desde su casa, la mayoría de los usuarios lo utilizan en el trabajo, en la escuela o en cibercafés (Instituto Nacional de Estadística y Geografía, 2008). Los miembros de las organizaciones a favor de los derechos sexuales y reproductivos tienen acceso a Internet debido a sus pertenencias a la clase media y a sus altos niveles educativos. La baja penetración de Internet puede afectar el diseño de los sitios como un instrumento de protesta virtual o como una esfera pública virtual, pero no afecta la interacción de los participantes del movimiento en el espacio virtual. El uso diario de los correos electrónicos y teléfono en línea como instrumentos principales de comunicación por parte de los participantes de este movimiento evidencia sus accesos a Internet.

El hecho de que menos de la mitad de los sitios web estudiados (9/20) ofrece la posibilidad de comunicación interactiva interna y externa - con la excepción de los grupos de protesta - , se debe al relativamente alto costo del conocimiento sobre el diseño de los sitios a nivel local por un lado y al incremento de las posibilidades de uso gratuito de las plataformas electrónicas al nivel global, por el otro.

El poco conocimiento sobre la tecnología de información digital y el alto costo de diseñar un sitio implican que la técnica del diseño de sitios está controlada por pocos profesionales en México (Schulz, 2002). Ninguna de las organizaciones o grupos entrevistados tiene a un miembro o personal que pueda diseñar o modificar directamente los contenidos de los sitios. Estos sitios son creados por un profesional de informática. De esta forma, integrar a las funciones interactivas significaría mayor costo en el diseño y en el mantenimiento del sitio web.

Los grupos de protesta, que no tienen recursos financieros utilizan el blog, una plataforma electrónica preparada y gratuita a nivel internacional, para subir información, fotos y para crear ligas y formas de comunicación. La función integrada de contestar a los mensajes o dejar comentarios en la plataforma prediseñada de un blog permite a los sitios web de los grupos de protesta tener una herramienta para la comunicación interna y externa.

En lugar de utilizar una plataforma preparada, los otros tres tipos de organizaciones (las coaliciones, organizaciones de los movimientos sociales y las organizaciones de apoyo) contratan a expertos técnicos para construir sus sitios. MEXFAM es una gran ONG con un departamento de diseño gráfico que esboza el contenido y las imágenes del sitio, pero el diseñador sólo concibe los gráficos y el contenido, mientras que la parte técnica se encargan los ingenieros de informática externos (entrevista-Delné). Otras organizaciones sin un departamento de diseño gráfico dependen más aún del soporte técnico externo. Esta dependencia técnica causa dificultades a las organizaciones en actualizar las informaciones de sus 
sitios por ellos mismos, sino para modificar el diseño o utilizar el sitio adecuadamente. Después, la integración de la herramienta de comunicación en los sitios requiere un diseño más complejo y mayor costo de mantenimiento. Esta dependencia técnica en el apoyo externo es un factor que interviene en el uso de los sitios de las organizaciones en México.

Por un lado, el control de los conocimientos de Internet por parte de unos pocos profesionales y el alto costo de estos conocimientos técnicos en el contexto local y por el otro, la disponibilidad global de las plataformas electrónicas gratuitas que ofrece posibilidades de comunicación interactiva, ambos factores juntos explican la falta de comunicación interactiva en los sitios web de la mayoría de las organizaciones con la excepción de los grupos de protesta.

\section{La burocracia de la financiación}

El requerimiento de apoyo financiero para las organizaciones es otro factor importante que afecta el uso de la tecnología. A mediados de 1990, 60\% de las ONG de mujeres dependían del apoyo financiero externo de donantes nacionales e internacionales (Tarrés, 1998). Los fondos internacionales para las organizaciones civiles mexicanas comenzaron a incrementarse después de la Conferencia Internacional de la Población que se llevó a cabo en la Ciudad de México en 1984. Muchas de las agencias internacionales en esta conferencia vieron las necesidades financieras para la implementación de programas de planeación familiar y de salud en México. Además, el apoyo de agencias multilaterales o bilaterales se usó para aliviar las secuelas del temblor en la Ciudad de México en 1985. La ayuda financiera extranjera y las movilizaciones locales para obtener servicios sociales y actividades motivaron la creación de las ONGs para la salud y de derechos humanos.

Gran parte de las actividades y proyectos de las organizaciones a favor de los derechos sexuales y reproductivos hoy en día depende de los recursos financieros internacionales y nacionales. Aparte de los grupos de protesta que rara vez reciben apoyo financiero, otros tipos de organizaciones utilizan parte de los fondos para construir sus sitios. ${ }^{2}$

El apoyo financiero de las agencias nacionales o internacionales de desarrollo conlleva procedimientos complejos de solicitar proyectos, escribir reportes y hacer evaluación de las actividades. Las coaliciones tienen difícilmente el tiempo y el esfuerzo necesarios para cumplir este proceso de obtener fondos.

Las cinco coaliciones estudiadas se crearon con diferentes propósitos y bajo circunstancias especiales. DEMYSEX es una red de organizaciones para el intercambio de información y actividades. Jóvenes por Nuestros Derechos y Tú Puedes Salvar la Vida son alianzas estratégicas para abogar y promover los derechos reproductivos. Alianza Nacional para el Derecho a Decidir (ANDAR) fue creada por los movimientos feministas en la Ciudad de México para evitar la aprobación de la ley de antiaborto en diferentes Estados. También existe una coalición como proyecto de cooperación entre diferentes organizaciones, El Ring de las Ideas. En todos estos casos, los fundadores solicitaron financiamiento a las fundaciones nacionales o agencias internacionales y recibieron apoyos financieros para construir un sitio web propio.

Solicitar proyectos, escribir reportes y ser evaluados son procedimientos que pertenecen al proceso burocrático de la política de financiamiento internacional y nacional. El hecho de escribir solicitud a fin de obtener fondos para una coalición como un proyecto colectivo es un trabajo adicional a las actividades regulares para los sus miembros organizaciones. Buscar apoyo financiero pertenece a las actividades principales de las ONG mexicanas para mantener sus actividades en marcha, pero escribir un proyecto para preservar los fondos de una coalición, a favor de otras organizaciones requiere un esfuerzo extra. Debido a la poca suma e inestabilidad de los fondos para las coaliciones, sus sitios web son muy simples y no provee posibilidad de comunicación interna o externa. A largo plazo, los miembros no son capaces de mantener y actualizar los sitios con sus propios recursos. Esta es la razón por la cual los sitios de las coaliciones ofrecen muy poca información y no pueden servir como una plataforma para la construcción de una comunidad del movimiento.

Otro factor que interviene en la construcción del sitio web son los requerimientos de evaluación periódica una vez que la coalición recibe el financiamiento. Las actividades de la red o coalición para estimular la movilización y construir la identidad colectiva entre los participantes son difíciles de comprobar y de ser evaluadas con criterios objetivos. La mayoría de los fondos apoyan a la iniciación de la coalición con un sitio web, pero no su mantenimiento permanente. Sin solicitar nuevos proyectos con otras metas y actividades concretas a parte de formar una comunidad de intercambio y formación de redes, las coaliciones no podrían obtener más recursos financieros.

El Ring de las Ideas es una excepción porque realiza proyectos concretos. Esta coalición promueve los derechos sexuales y reproductivos entre los jóvenes al montar obras de teatro cortas en las calles y recibe financiamiento continuo por parte de agencias internacionales de desarrollo. Para anunciar sus actividades en línea, el costo de su sitio web fue cubierto 
por los fondos y, por lo tanto, su sitio estaba sujeto a la supervisión de los evaluadores de las agencias internacionales. Luego de revisar los contenidos de su sitio web y los reportes de sus actividades, los evaluadores hacen recomendaciones de las modificaciones necesarias para el sitio. El sitio web sirve como una herramienta de monitoreo a larga distancia sobre el cumplimiento del proyecto (Entrevista-Diana).

Tarrés (2001) señaló que la dependencia financiera de las ONG de mujeres de los fondos internacionales llevaba a que las agencias externas tuvieran el control de la agenda, la organización y las actividades de estas ONGs. La observación de los sitios web confirma la observación de Tarrés y demuestra la intervención de los fondos externos en el uso de Internet de las organizaciones.

\section{La relación con los medios tradicionales}

Una función importante de Internet es la transmisión de opiniones críticas en una esfera pública alternativa a los medios de comunicación tradicionales. En este sentido, Internet es una herramienta para que el movimiento social interviene en la esfera pública dominada por los medios tradicionales.

Proporcionar noticias en sus sitios web es un rasgo específico de las organizaciones mexicanas. Doce de los veinte sitios reproducen las noticias originadas en los medios tradicionales de comunicación en la forma de transcripciones o ligas a la fuente original, en lugar de ofrecer noticias independientes en el espacio virtual. Contrario a la expectativa de que Internet sea una fuente alterna de noticias, los sitios de estas organizaciones mexicanas sirven como un instrumento para difundir las noticias que ofrecen las prensas existentes.

Si Internet es considerada como un instrumento "alternativo" de comunicación en relación con los medios tradicionales de comunicación, entonces la estructura de estos últimos que favorece o restringe al movimiento social debería ser considerada en el análisis de los nuevos medios. Desde esta perspectiva, hay dos factores que podrían explicar el fenómeno de la reproducción de noticias en los sitios de las organizaciones estudiadas.

La primera razón es que el uso de los medios tradicionales como instrumento estratégico para el movimiento feminista lleva a muchas organizaciones mexicanas a tener su propia estación de radio, revista o boletín de noticias. Tres de las organizaciones estudiadas - que son más jerárquicas y tienen más recursos financieros - pertenecen a esta categoría. Salud Integral para la Mujer A.C. y Católicos por el Derecho a Decidir tienen fuentes periodísticas propias desde los
80, las mismas noticias al aire o en boletines se presentan también en línea. Letra $S^{3}$ - otra organización especializada en información y periodismo en el área de los derechos reproductivos y sexuales - publica regularmente un suplemento para el periódico La Jornada, y tiene una nueva agencia, Notiese, especializada en cuestiones sexuales y reproductivas. Debido a que estas organizaciones utilizan los medios tradicionales propios para sus movilizaciones, Internet no se considerará como un espacio "alterno", sino como un instrumento de más para difundir la información y noticias de los periódicos, revistas o la radio.

La segunda razón es que el tema de salud sexual y reproductiva no son marginales en los medios tradicionales. El Excelsior, La Jornada y su suplemento femenino son periódicos que incluyen perspectivas feministas en sus publicaciones. México tiene una organización periodística feminista - Comunicación e Información de la Mujer A.C. (CIMAC - que coopera con más de 30 periódicos locales nacionales y prensas feministas internacionales. Esta organización fue fundada en 1988 por Sara Lovera y Yoloxochitl Casas, quien también construyó la Red Nacional de Periodistas - una red que reunía a periodistas feministas de México y otros países de Latinoamérica y el Caribe (Quintero, 2004).

Estos echos indican una estrecha relación entre el movimiento feminista y el periodismo en México que construye un contexto favorable para el movimiento sexual y reproductivo que abundan de boletines, revistas, programas de radio y centros noticieros feministas, tales como CIMAC y Notiese.

En este contexto, las organizaciones del movimiento no crean los sitios con el propósito de brindar información independiente, sino para difundir las noticias existentes de los medios de comunicación tradicionales dentro de la comunidad del movimiento o el público. El movimiento de los derechos sexuales y reproductivos utiliza la nueva tecnología como un instrumento complementario a los medios tradicionales.

\section{Conclusión}

El análisis de los sitios web de las organizaciones de los movimientos por los derechos sexuales y reproductivos en la Ciudad de México pone en evidencia que el enfoque racional-instrumentalista no es suficiente para explicar el diseño de estos sitios. Por ejemplo, los sitios de las coaliciones brindan menos posibilidad de comunicación interactiva que los grupos de protesta, y los sitios de los movimientos repro- 
ducen noticias provenientes de los medios tradicionales de comunicación. Estos dos hechos contradicen el determinismo de la racionalidad de las organizaciones en el uso de Internet. Los resultados de entrevistas muestran que más allá de los rasgos organizacionales, el contexto sociopolítico de las organizaciones juega un papel importante en el diseño de los sitios.

$\mathrm{La}$ infraestructura global de Internet que interactúa con el monopolio local del conocimiento tecnológico conduce a un fenómeno peculiar: los sitios de las organizaciones con más recursos no proveen comunicación interactiva, pero los de pocos recursos sí pueden. El diseño de los sitios está contextualizado por los factores globales y locales, y aquí reside la posibilidad ofrecida por Internet para los grupos con pocos recursos de superar la limitación al nivel local.

La dependencia de los fondos externos y el proceso burocrático por parte de las agencias internacionales de desarrollo hace que el mantenimiento regular de los sitios sea difícil y, al mismo tiempo, la evaluación de lo sitios como parte del proyecto de desarrollo constriñe el diseño y el contenido de los sitios para asumir los consejos externos.

\section{Agradecimiento}

Este studio fue financiado por National Science Council of Taiwan (NSC 97-2410-H-305-031MY2). Una version previa fue presentada en el XVII Congreso Mundial de Sociologia en Gothenburg,
La relación de un movimiento social con los medios masivos de comunicación afecta el diseño de los sitios. La estrecha relación entre el feminismo y el periodismo en México lleva a las organizaciones estudiadas a utilizar Internet como un instrumento para difundir las noticias de los medios tradicionales, sus sitios se convierten en una extensión de estos medios masivos tradicionales, en lugar de ser nuevos espacios para crear noticias críticas alternativas.

El uso de Internet por parte de los movimientos sociales está contexualizado en su localización geográfica, en la que influyen factores sociales, económicos, politicos y culturales. Este artículo revela la importancia de tres factores para la construcción de los sitios web de estos movimientos en la Ciudad de México: el conocimiento tecnológico, la dependencia financiera de las agencias de desarrollo internacionales y la relación con los medios tradicionales. El acceso pleno a Internet no implica simple uso igualitario de este instrumento, para un mejor entendimiento del uso de Internet y los efectos de las nuevas tecnologías digitales para los movimientos sociales, se require análisis de sus contextos sociopolíticos en el futuro.

Suecia, 11-17 de julio de 2010. La autora agradece especialmente a las personas entrevistadas en la Ciudad de Mexico. El presente trabajo no sería posible sin sus colaboraciones.

\section{Referencias}

BENNETT, W. Communicating global activism. Information, Communication \& Society, no 6, v. 2, p. 143-168, 2003.

BECKLES, Colin, Black liberation and the internet. A strategic analysis. Journal of black Studies, $n^{\circ} 31$, v.3, p. 311324, 2001.

CARDACI, Dora. Salud, género y programas de estudios de la mujer en México. México D.F.: UNAM - OPS - UAM, 2004. DELLA PORTA, Donatella; MOSCA, Lorenzo. Searching the net. Information, Communication \& Society, n'12, v. 6, p. 771-792, 2009.

DIANI, Mario. Social Movement Networks: Virtual and Real. In: WEBSTER, F (Ed.) Culture and Politics in the Information Age. New York:Routledge, 2001. p.117-128. EDWARDS, Arthur. The Dutch Women's Movement On-line: Internet and the Organizational Infrastructure of a Social Movement. In: WIN VAN de DONK, B; LOADER, P. Nixon; RUCHT, D. (Eds) Cyberprotest: New Media, Citizens and Social movements. London and
New York: Routledge, 2004. p. 183-206.

GANESH, S.; BARBER, K. The silent community: organizing zones in the digital divide. Human Relations, $\mathrm{n}^{\circ}$ 62, v.6, p. 851-874, 2009.

FENOLLOSA, Ligia Tavera. Nuevas Tecnologías y Organizaciones Civiles: El Caso de LaNeta. Perfiles Latinoamericanos, no 18, p. 59-74, 2001.

FROEHLING, Oliver. The Cyberspace War of Ink and Internet in Chiapas, Mexico. Geographical Review, $\mathrm{n}^{\circ}$ 87, v. 2, p. 291-307, 1997.

GRABER, Doris A.; BIMBER, Bruce; BENNET, W. Lance; DAVIDS, Richard; NORRIS, Pippa. The Internet and Politics: Emerging Perspectives. In: NISSENBAUM, Helen and PRICE, Monroe E. (Eds.) Academy and the Internet. New York: Peter Lang, 2004. p. 90-119.

INEG (Instituto Nacional de Estadística y Geografía). Estadísticas a propósito del Día Mundial de Internet. Datos nacionales. Instituto Nacional de Estadística y Geografía, México, 2009. 
KULCZYCKI, Andrezej. The Abortion Debate in Mexico: Realities and Stalled Policz Reform. Bulletin of Latin American Research, no 6, v. 1, p. 50-68, 2007.

ORTIZ-ORTEGA, Adriana y BARQUET, Mercedes. Gendering transition to democracy in Mexico. Latin American Research Review, Special Issue, 2010, v. 45, p. 108-137, 2010.

PUDROVSKA, Tetyana, and FERREE, Myra Marx. Global Activism in 'Virtual Space': The European Women's Lobby in the Network of Transnational Women's NGOs on the Web. Social Politics, $n^{\circ} 11$, v.1, p.117-143, 2004.

QUINTERO, Genoveva Flores. Prensa feminista: 30 años de batallas por el espacio público. In: GOSSIO, María Elena García (Ed.) Mujeres y Sociedad en el México contemporáneo. Nombrar lo innombrable. Monterrey: Tecnológico de Monterrey, 2004. p. 203- 231.

RHEINGOLD, Howard. From the Screens to the Streets, The Extreme Democracy: Book and Discussion Forum for Networked Activists. 2003. http://www.extremedemocracy. com/chapters/Chapter\%20Seven-Rheingold.pdf Viewed 27 September 2009.
SCHULZ, Markus. Internet und Politik in Lateinamerika: Mexiko. Frankfurt am Main: Vervuert Verlag, 2002.

SCHULZ, Markus. The role of the internet in transnational mobilization. A case study of the Zapatista Movement, 1994-2005. In: HERKENRATH, Mark (Ed.) Civil society: local and global responses to global challenges. Berlin: Lit. Verlag, 2007. p. 129-156.

STEIN, Laura et al. Social Movement Web Use in Theory and Practice: a Content Analysis of US Movement Websites. New Media E Society, no 11, v. 5, p.749-771, 2009. TARRÉS, María Luisa. De la identidad al espacio público: las organizaciones no gubernamentales de mujeres en México. In: MENDEZ, José Luis (Ed.) Organizaciones civiles y políticas públicas en México y Centroamérica. México: Miguel Angel Porrúa, 1998.

TARRÉS, María Luisa. Las organizaciones del movimiento de mujeres en la reforma política. In: OLVERA, Alberto J. (Ed.) La sociedad civil: De la teoría a la realidad. México: El Colegio de México, 2001.

\title{
Analysis of sexual and reproductive rights movement in Mexico websites
}

\begin{abstract}
This article analyzed sexual and reproductive rights movement in Mexico City websites focusing on explaining the discrepancy between the internet potential and its real use, and on how the sociopolitical context, through digital means, has shaped the social movements' organizational strategies. The research design entailed two procedures: analysis of 20 organizations' websites and semi-structured interviews with 19 activists. The results showed that in spite of organizative features, the knowledge on information technology, the burocracy in the funding process, and the relation with traditional means of communication are factors that interfere in the way the sexual and reproductive moment in Mexico uses the internet.
\end{abstract}

Key words: internet, social movement, sexual and reproductive rights, Mexico.

\section{Análise dos sites do movimento pelos direitos sexuais e reprodutivos no México}

\section{Resumo}

O artigo analisa os sites do movimiento pelos direitos sexuais e reprodutivos na Cidade do México com o objetivo de explicar a discrepância entre a potencialidade da internet e seu uso real; e analisar como o contexto sociopolítico influencia as estratégias de organização dos movimentos sociais pelos meios digitais. O projeto de pesquisa possui dois procedimentos: análise dos 20 sites das organizações e entrevistas semiestruturadas com 19 ativistas. O resultado mostra que, apesar das características organizativas, o conhecimento sobre as tecnologias da informação em geral, a burocracia no proceso de financiamento e a relação com os meios tradicionais de comunicação, são fatores que interferem no uso da Internet por parte das organizações de direitos sexuais e reprodutivos no México.

Palavras-chave: Internet, movimento social, direitos sexuais e reprodutivos, México.

Data de recebimento do artigo: 01/11/2011

Data de aprovação do artigo: 29/01/2012 
Apéndice 1. Los sitios web de las organizaciones por derechos sexuales y reproductivos analizadas

\begin{tabular}{|c|c|c|}
\hline Organisation's Name & Website & Tipo de organización \\
\hline JÓVENES NUESTROS DERECHOS & http://www.jovenesnuestrosderechos.org/ & Coalición \\
\hline DEMYSEX & http://www.demysex.org.mx/Demysex/Inicio.html & Coalición \\
\hline EL RING DE LAS IDEAS & http://elringdelasideas.spaces.live.com/ & Coalición \\
\hline ANDAR & http://www.andar.org.mx/ & Coalición \\
\hline TÚ PUEDES SALVAR LA VIDA & http://www.tupuedessalvartuvida.org/vida & Coalición \\
\hline COLECTIVA CON LETRA F & http://colectivaconletraefe.blogspot.com/ & Grupo de protesta \\
\hline GLUNAM & http://glunam.blogspot.com & Grupo de protesta \\
\hline ROSA CHILLANTE & http://rosachillante.blogspot.com/ & Grupo de protesta \\
\hline DECIDIR & http://www.decidir.org.mx/ & Grupo de protesta \\
\hline $\begin{array}{l}\text { CATÓLICAS POR EL DERECHO A } \\
\text { DECIDIR }\end{array}$ & http://www.catolicasmexico.org/ & $\begin{array}{l}\text { Organización de movimiento } \\
\text { social }\end{array}$ \\
\hline ELIGE & http://www.elige.net/ & $\begin{array}{l}\text { Organización de movimiento } \\
\text { social }\end{array}$ \\
\hline EQUIDAD DE GÉNERO & http://www.equidad.org.mx/nuevo/entradadef.htm & $\begin{array}{l}\text { Organización de movimiento } \\
\text { social }\end{array}$ \\
\hline BALANCE & http://www.redbalance.org/index.htm & $\begin{array}{l}\text { Organización de movimiento } \\
\text { social }\end{array}$ \\
\hline SIPAM & http://sipam.org.mx/ & $\begin{array}{l}\text { Organización de movimiento } \\
\text { social }\end{array}$ \\
\hline HOMBRES POR LA EQUIDAD & http://www.hombresporlaequidad.org.mx/ & Organización de apoyo \\
\hline LETRA S & http://www.letraese.org.mx/ & Organización de apoyo \\
\hline GIRE & http://www.gire.org.mx/ & Organización de apoyo \\
\hline MEXFAM & http://www.mexfam.org.mx & Organización de apoyo \\
\hline SEMILLAS & http://www.semillas.org.mx/ & Organización de apoyo \\
\hline ABORTOS & http://www.abortos.com.mx/ & Organización de apoyo \\
\hline
\end{tabular}

